International Journal of Wireless \& Mobile Networks (IJWMN) Vol. 4, No. 1, February 2012

\title{
EFFECT OF MULTIPATH FADING AND Propagation ENVIRONMENT ON THE Performance of a Fermat Point Based ENERGY EFFICIENT GEOCAST ROUTING PROTOCOL
}

\author{
Kaushik Ghosh $^{[1]}$, Partha Pratim Bhattacharya ${ }^{[2]}$ and Pradip K Das ${ }^{[3]}$
}

Faculty of Engineering \& Technology, Mody Institute of Technology \& Science

(Deemed University), Lakshmangarh, Dist. Sikar, Rajasthan - 332311, India

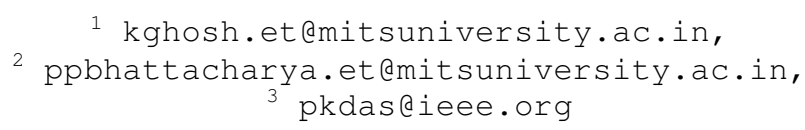

\section{ABSTRACT}

Energy efficiency is a much talked about thing in the domain of geocast routing protocols for Wireless Ad Hoc and Sensor Networks (WASNs). Fermat point based protocols are capable of reducing the energy consumption of a WASN by reducing the total transmission distance in a multi hop-multi sink scenario. Presently, there are quite a handful of them but many of them have not considered the effect of changing propagation environment around the considered network while measuring the performance of the protocol. Congested environment around a WASN increases the chance of multipath propagation and it in turn introduces multipath fading. In this paper, the effects of both of these factors are considered on the performance of I-Min routing protocol designed for WASNs.

\section{KEYWORDS}

WASN, Geocasting, Energy efficiency, Multi path fading, Propagation environment.

\section{INTRODUCTION}

Geocast routing protocols have become an integral part in the domain of routing for Wireless Ad Hoc and Sensor Networks (WASNs). It is well known that these kind of networks demand special attention towards energy consumption due to their inherent nature and places of their deployment. Energy is in fact a function of the inter nodal distances $\left(\mathrm{d}^{\mathrm{n}}\right)$, where $\mathrm{n}$ is called path loss exponent. Quite a few protocols of said type have been proposed over the years by numerous authors but the radio models used in most of them have undermined the effect of changing propagation environment around the network while determining its energy expenditure. A hullabaloo around a WASN is sure to introduce multipath fading, which again demands some modification in the radio model proposed for a free space condition. In this paper we have considered both these parameters to find out the degree of variation on the performance of a protocol that doesn't consider either of these two parameters. Here, the geocast routing protocol under consideration is the I-MIN protocol [1]. In [1] the radio model used considered data size and distance between the nodes as the functions of energy consumed by a node while transmitting. Energy consumed by a node while receiving was however a function of the received data volume only. Not only this, the network was assumed to be operating in a free space only $(n=2)$, as was considered by many other protocols [2], [3], [4], 
International Journal of Wireless \& Mobile Networks (IJWMN) Vol. 4, No. 1, February 2012

[5].In this paper we have modified the radio model used in [1] by incorporating both of the parameters. From Frii's free space equation we get

$$
P_{r}=P_{t} *\left(G_{t} * G_{r} * \lambda^{2}\right) /\left(16 * \pi^{2} * d^{2} * L\right)
$$

Here, $P_{r}$ and $P_{t}$ are the power required for receiving and transmitting respectively over a distance of $\mathrm{d}$. So it is clear that the power consumed by a node while receiving data is dependent upon the received signal strength from the sender along with the distance between them. Now, in order to include that factor in $\mathrm{P}_{\mathrm{r}}$, it is not sufficient to know $\mathrm{P}_{\mathrm{t}}$ alone. Because multipath fading [6] causes the signal strength at the receiving ends to decrease by some order which cannot be predicted using the above equation alone. The nature of multipath fading is not as simple as an exponential decay shown in most of the elementary text books. Findings in [6] shows the signal strength to decrease as a result of multipath fading with distance but the nature of the graph is bit complicated. In this paper we have thus tried to modify the radio model in [1] with the findings of [6] and changing the values of $n$ to all integer values between 2 and 6 . Coming to the distance part, it becomes evident that we need to find a way or the other to reduce the total transmitting distance where the nodes seldom changes their deployed position. A Fermat point based scheme helps the most in this regard. There are however techniques other than Fermat point based ones for reducing $d$ and thereby minimizing the energy expenditure of a WASN.

The remaining sections include related works (2), a brief discussion of the I-MIN protocol (3), effect of changing propagation environment around the network and multipath fading on that protocol (4), results(5) and finally, conclusion and future works(6).

\section{RELATED WORKS}

The introduction of Fermat point based routing in geographic routing protocols had been made for the first time in [7]. In [7] we get a detailed discussion on locating the Fermat point for a triangular region in a geometric way. [3] have shown to do the same by finding the Global Minima which ensures a more generalized approach of finding the Fermat point for any number of geocast regions. However, the forwarding technique in [3] couldn't ensure a loop free solution. This problem was eliminated in [1] and the results were thus better when it came to energy consumption and total number of hops encountered by a data packet before it reached its final destination. Exploiting the concept of Fermat point for energy reduction is done in [8] as well. Data dissemination for multi sink scenario through Fermat point is again shown in [9] using a graph based approach. In fact Fermat point based approach has been used by the authors since it is capable of reducing the transmission distance in a multi sink-multi hop environment. However, many other techniques other than the using the Fermat point for distance reduction has also been explored. Of all the different techniques, Location Aided Routing (LAR) [10] can be termed as the ancestor of many of the present day geocast routing protocols. LAR professed for reducing the number of transmissions by determining the geographic region of the destination. However, action on encountering routing holes was unknown till authors in [11] proposed the idea of perimeter routing for the same. Reducing the distance to reduce the energy consumed through a non Fermat point based approach has been shown in [12] as well. Although outdated, [13] gives a detailed survey on geocast routing protocols. [14] on the other 
International Journal of Wireless \& Mobile Networks (IJWMN) Vol. 4, No. 1, February 2012

hand most probably have introduced the concept of geocasting itself in the field of MANETs. The aforementioned works have mainly focused on reducing the transmission distance between the nodes without disturbing their initial deployment to attain an energy friendly nature.

Techniques other than distance reduction for energy consumption have also been used in WASNs [15-18]. But of all the protocols discussed, none have discussed the effect of changing propagation environment or multipath fading on the amount of energy consumed by the network.

In this paper we have discussed how change in propagation environment around a WASN can affect the performance of energy efficient routing protocols, even when all other factors remain unchanged.

\section{I-MIN SCHEME}

The I-Min scheme has been described in [1] in details, yet for sake of continuity let us re describe it in brief. The said scheme uses a Fermat Point based data forwarding technique [1-3], [7]. It is a protocol designed for static WASNs with a number of geocast regions to transmit a message to. The Global Minma algorithm [3] is used for finding out the Fermat point of the polygonal/triangular region under consideration (figure 1a). The results in [1] have shown it to outperform some of its predecessors like [2] and [3] when energy and delay are the measuring parameters (Fig 1). The reason for a stark difference in performance between I-MIN and its predecessors are due to the fact that in neither of them the authors have talked about a loop free solution which in fact, I-MIN did.

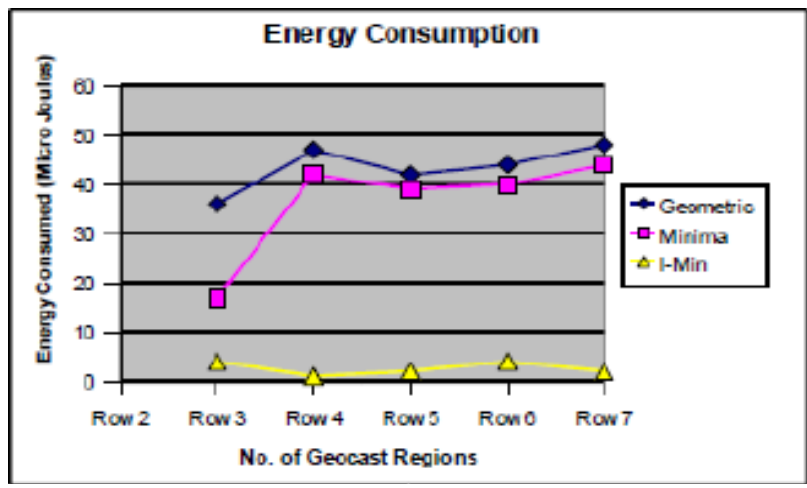

Fig 1a. Energy consumption in the three different schemes.

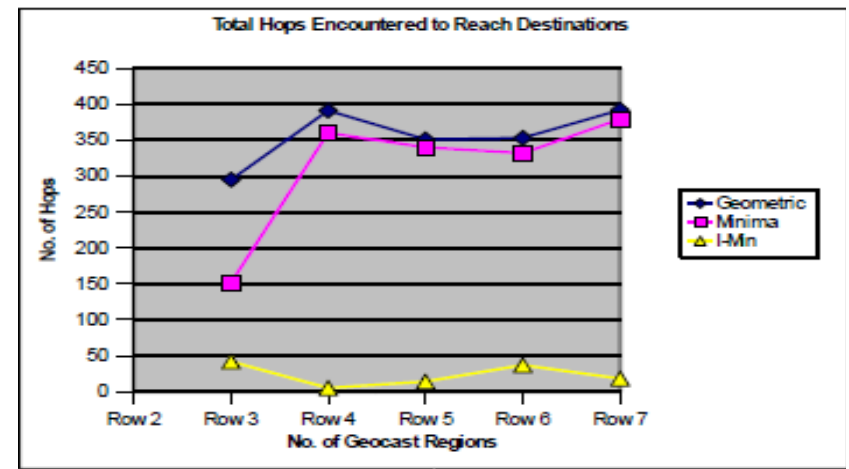

Fig 1b. Comparison between three schemes with respect to number of hops encountered by a packet to travel from a given source to destination. 
International Journal of Wireless \& Mobile Networks (IJWMN) Vol. 4, No. 1, February 2012

The protocol under consideration uses a localized greedy approach while forwarding data (Fig 2b) i.e. a node forwards a packet to such a node amongst its neighbors which is closest to the destination. Although, one of the major problems of this kind of an algorithm is local maximization [11], yet we expect it not to happen in our case as the network under consideration has been taken dense enough to have a hole in it. Moreover, another improvement over the forwarding method of I-Min scheme has been made here.

The next forwarding node here is selected not merely on the basis of its distance from the destination but its residual energy has also been taken into consideration. In figure $1 \mathrm{~b}$ we see that the next node is selected from a list of neighbors by comparing their distance from the destination. In this paper we would like to modify the forwarding technique through the following equation

max_id =res_energy $* u_{1}-d i s t * u_{2}$.

The parameter max_id is calculated for all the neighbors of a node and the one with highest value of $\boldsymbol{m a x} \boldsymbol{i} \boldsymbol{i} \boldsymbol{d}$ becomes the next forwarding node.

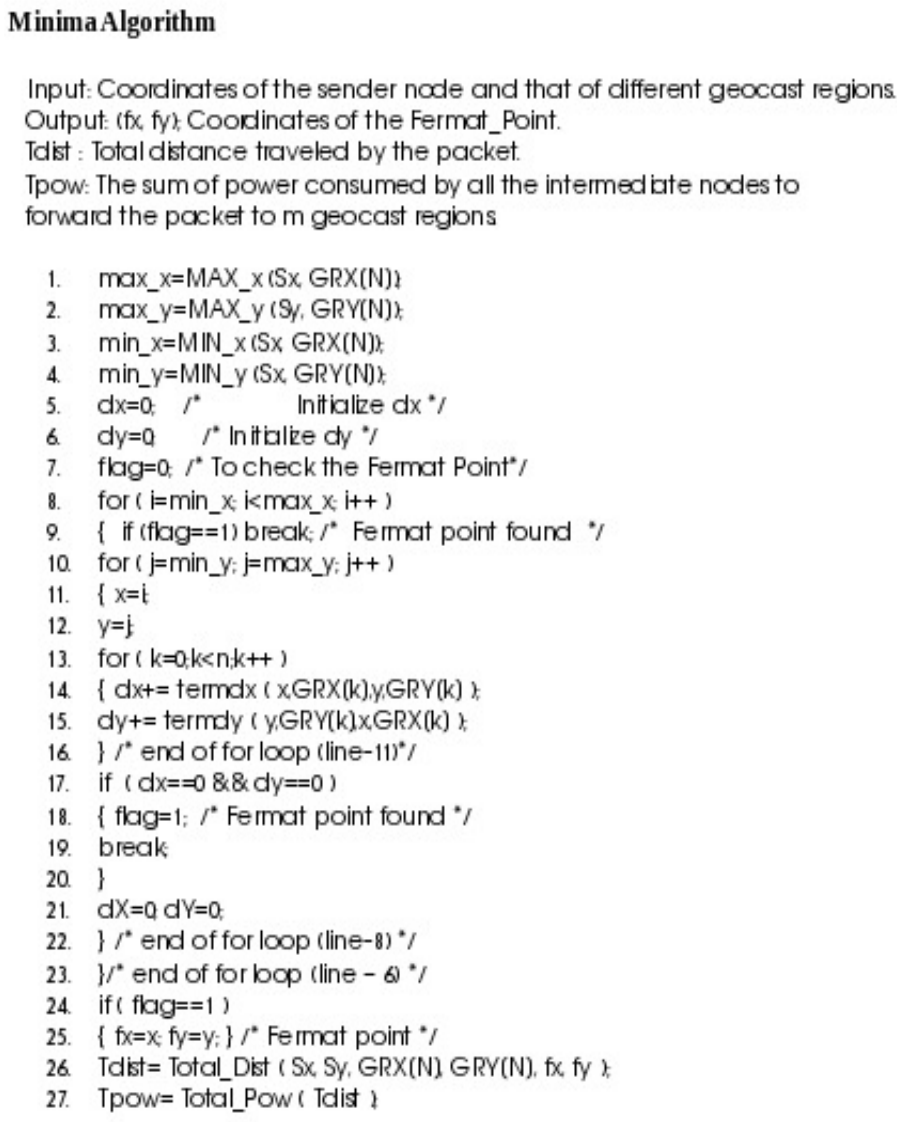

Figure 2a. Global Minima Algorithm. 


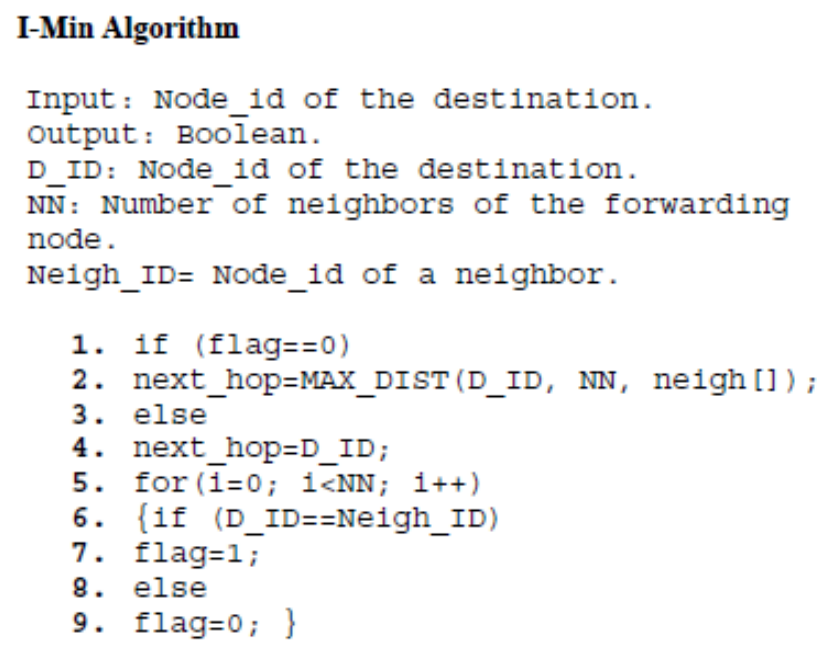

Figure 2b. I-MIN forwarding scheme.

Here, res_energy is the residual energy of a node and dist is its distance of the node from the destination or Fermat point. This is because, for a node lesser the distance from destination and higher the residual energy, greater is its potential to be the next forwarding node amongst a group of nodes. Both these parameters are known to a node when a neighbor piggybacks them with data packets in case there is any change in those parameters. $\boldsymbol{u}_{1}$ and $\boldsymbol{u}_{2}$ are two weight age functions where $\boldsymbol{u}_{1}>\boldsymbol{u}_{2}$. This way we make the scheme energy efficient as it increases the probability that a node with higher residual energy is selected even if its distance from destination is somewhat more as compared to that for another node with a lesser value for residual energy.

\section{Effect of Propagation Environment and Fading}

In this section we will consider separately the effect of propagation environment and multipath fading on the radio model used in [1] and then would combine them to present a new radio model to be used in WASNs.

\subsection{Effect of Propagation Environment}

The radio model in [1] has considered the space between the constituent nodes of the network to be free space only. In fact it is the model used in [5] as well. The model is given below for clarity

$$
\begin{aligned}
& E_{T X}(m, d)=m * E+m * \epsilon * d^{2} \\
& E_{R X}(m)=m * E
\end{aligned}
$$

Where,

$E=50 \mathrm{~nJ} / \mathrm{bit}$ and $\varepsilon=10 \mathrm{pJ} / \mathrm{bit} / \mathrm{m}^{2}$.

$E_{T X}=$ Energy consumed for transmission.

$E_{R X}=$ Energy consumed for reception.

$d=$ distance between the transmitting and receiving node.

$\varepsilon=$ Permittivity of free space.

$m=$ Number of bits. 
However, the scenario in real life applications may not be that simple. Value of path loss exponent $\mathrm{n}$ may range from 2 for free space to 6 for congested city. We have thus taken a whole range of values considering $n=2,3,4,5$ and 6 . At every step we find the energy to increase in the order of 100. Thus it becomes evident how much more the distance between the nodes is going to affect the performance of a protocol when the same experiment is repeated in a city rather than in rural area or free space.

We are thus generalizing the above model as

$\mathrm{E}_{\mathrm{TX}}(\mathrm{m}, \mathrm{d})=\mathrm{m} * \mathrm{E}+\mathrm{m} * \dot{\varepsilon}^{*} \mathrm{~d}^{\mathrm{n}}$

$\mathrm{E}_{\mathrm{RX}}(\mathrm{m})=\mathrm{m} * \mathrm{E}$.

With all other things retaining their meanings, $\dot{\varepsilon}$ is permittivity of air as medium.

\subsection{Effect of Fading}

In this section we modify the radio model of [1] yet further. Other than effect of propagation environment, what is going to have an impact on the performance of energy aware protocols, is the fading due to multipath propagation of the signals. In practical systems, hardly there is any LOS link and we have to depend on multipath signals. The signal strengths from two transmitting nodes in a multipath fading environment are given in Fig 3[6].

From the results in [6] we get the received signal strength at a node to be $-50 \mathrm{dBm}$ when the transmitting node is 100 meters away. Since the typical transmitting range of a sensor node is 100 meters or so, we include this $-50 \mathrm{dBm}$ of signal strength in the receiver ends to modify our radio model. To precise, the distance of a forwarding node from its immediate predecessor is always taken as 100 meters for the sake of simplicity. Because, otherwise things would get complicated since at every hop, the said distance varies arbitrarily between any values within 100 meters.

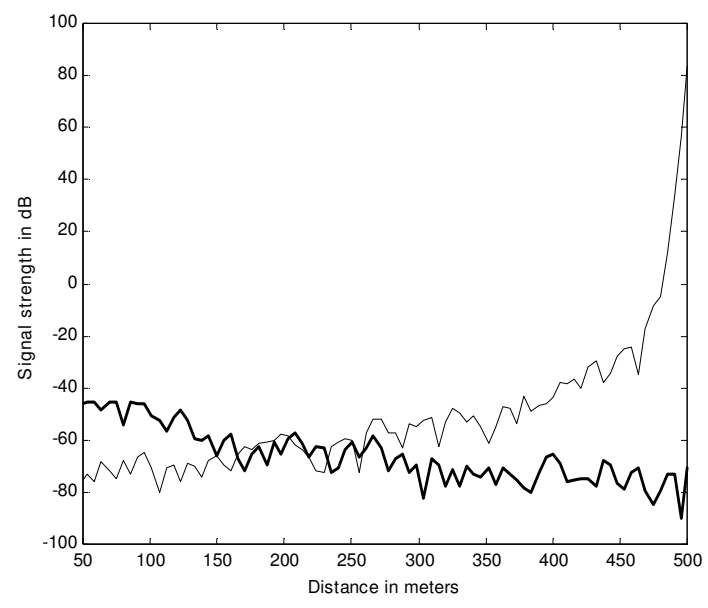

\section{Node A}

\section{Node B}

Fig 3. Received signal strength.

So, if we try to make that consideration, it won't be possible to include the effect of multipath fading in the radio model. The new radio model thus turns out to be

$P_{T X}\left(m^{\prime}, d\right)=m^{\prime} * E+m * \dot{\varepsilon}^{*} d^{n}$

$P_{R X}(m)=m * E+10^{-5}$. 
Where,

$\boldsymbol{P}_{T X}=$ Power required for transmission

$\boldsymbol{P}_{R X}=$ Power received at the receiver

$\boldsymbol{m}=$ Data rate

$\dot{\varepsilon}=$ Permittivity

$\boldsymbol{d}=$ Distance between nodes

$\boldsymbol{n}=$ path loss exponent whose value lies between 2 to 6 .

The factor $10^{-5}$ accounts for the amount of received power at the receiver's end (-50 dB), when the distance from transmitter is 100 meters (see figure 2). Since the typical transmitting range of a sensor node is 100 meters or so, we include this $-50 \mathrm{~dB}$ of signal strength in the receiver ends to modify our radio model. To be precised, the distance of a forwarding node from its immediate predecessor is always taken as 100 meters for the sake of simplicity.

\section{RESULTS}

The results here show that after modifying the radio model of [1] with considerations for changed propagation environmental effects and multipath fading, the consumption of energy in a geocast routing protocol will vary considerably (Fig 4). Figure 4 shows the condition when the numbers of geocast regions are only 3 . Higher the number of geocast regions, larger is the total distance that a data packet has to travel and thereby greater is the effect of propagation environment combined with the effect of multipath fading on the performance of an energy aware algorithm. This condition is depicted in figure 4.

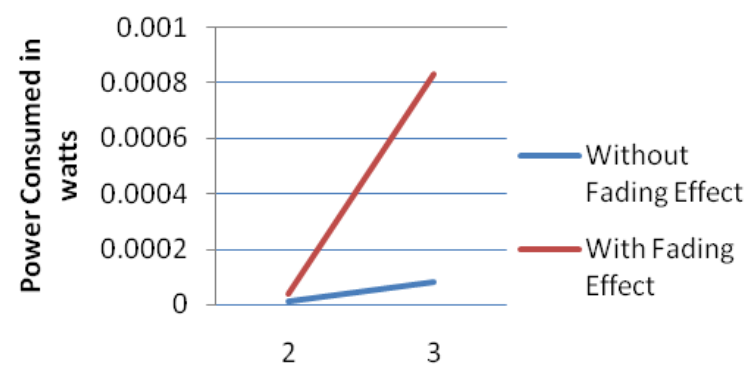

Different Values of $n$

Fig 4a. Effect of propagation environment and multipath fading on energy consumption $(\mathrm{n}=2$ and 3)

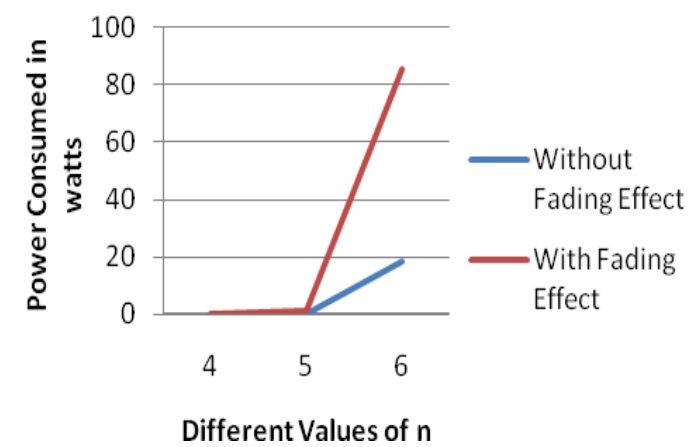

Fig 4 b). Effect of propagation environment and multipath fading on energy consumption

$$
(\mathrm{n}=4,5 \text { and } 6)
$$


The figure above depicts that the energy consumption of a network increase considerably when the value of $n$ reaches 6 . Although that is an extreme case, yet the results have shown that even a moderate value of $\mathrm{n}$ (say 4 , which is most common) the energy consumption of a network is to increase by an order of 1000 when compared to the same network under free space condition $(n=2)$.

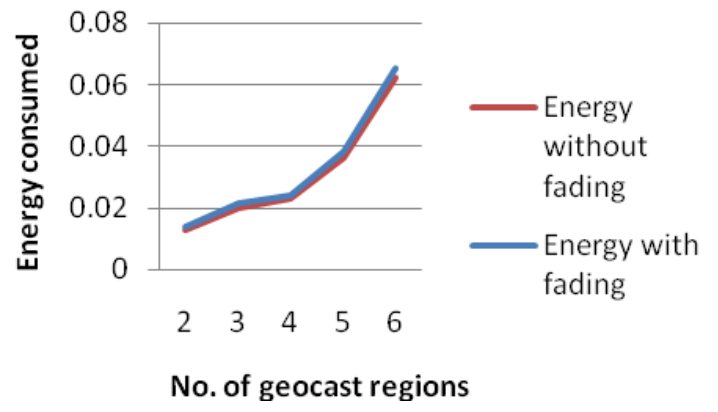

Fig 5. Energy consumption vs. number of geocast region $(n=4)$

From Figure 5 we find out that though small, but consideration of fading effect is increasing the energy consumption of a WASN when all other factors including the total number of hops encountered, remain the same.

\section{Conclusions}

Results in this paper are good enough to show that the effect of propagation environment and multipath fading are something which can never be ignored while forming a radio model for energy aware geocast routing protocols. The same protocol would consume larger amount of energy while operating in a congested environment than in a free space. As future work, one can think of a scheme where the effect of fading can be incorporated in the radio model for exact distance between the nodes than an approximated one as in the present paper. This in fact can be added on with the effect of pulsating data stream. The same amount of data received by a receiver over an intermittent period of time may result in better battery utilization than received in a single iteration. Modifying the protocol in that direction may put some further light on its behaviour.

\section{ACKNOWLEDGEMENTS}

The authors would like to acknowledge Mody Institute of Technology \& Science, Rajasthan, for providing immense support towards the present work.

\section{REFERENCES}

[1] Kaushik Ghosh, Sarbani Roy and Pradip K. Das "I-Min: An Intelligent Fermat Point Based Energy Efficient Geographic Packet Forwarding Technique for Wireless Sensor and Ad Hoc Networks", International Journal on Applications of Graph Theory in Wireless Adhoc Networks and Sensor Networks(GRAPH-HOC), Vol.2, No.2, June 2010 pp 34-44.

[2] S.H. Lee and Y.B. Ko. "Geometry-driven Scheme for Geocast Routing in Mobile Adhoc Networks”, IEEE Transactions for Wireless Communications, VTC Spring 2006: 638-642. 
International Journal of Wireless \& Mobile Networks (IJWMN) Vol. 4, No. 1, February 2012

[3] Kaushik Ghosh, Sarbani Roy and Pradip K. Das, "An Alternative Approach to find the Fermat Point of a Polygonal Geographic Region for Energy Efficient Geocast Routing Protocols:Global Minima Scheme”, AIRCC/IEEE NetCoM 2009.

[4] W.B. Heinzelman, A.P. Chandrakasan and H. Balakrishnan, "An Application-specific Protocol Architecture for Wireless Micro Sensor Networks", IEEE Transactions on Wireless Communications, Vol.1, Issue 4, October 2002.

[5] I-Shyan Hwang and Wen-Hsin Pang, "Energy Efficient Clustering Technique for Multicast Routing Protocol in Wireless Adhoc Networks", IJCSNS, Vol.7, No.8, August 2007.

[6] P. P. Bhattacharya, P. K. Banerjee, "User Velocity Dependent Call Handover Management", International Journal HIT Transaction on ECCN, Vol 1, No. 3, pp 150-155, July 2006.

[7] Young-Mi Song, Sung-Hee Lee and Young-Bae Ko "FERMA: An Efficient Geocasting Protocol for Wireless Sensor Networks with Multiple Target Regions" T. Enokido et al. (Eds.): EUC Workshops 2005, LNCS 3823, pp. 1138 - 1147, 2005. (C) IFIP International Federation for Information Processing 2005.

[8] K-F Ssu, C-H Yang, C-H Chou and A-K Yang "Improving RoutingDdistance for Geographic Multicast with Fermat Points in Mobile Adhoc Networks", Computer Networks 53 (2009) pp 2663-2673.

[9] P-J Chuang and B-Y Li "Fermat Point Based Data Dissemination in Sensor Networks", Journal of the Chinese Institute of Engineers, Vol. 32, No. 7 pp. 959-966 (2009).

[10] Y.B. Ko and N. H. Vaidya, "Location-aided routing (LAR) in Mobile Ad Hoc Networks", ACM/Baltzer Wireless Networks (WINET) journal, vol. 6, no. 4, 2000, pp. 307-321.

[11] B.Karp and H.Kung, "GPSR: Greedy perimeter stateless routing for wireless networks", ACM/IEEE MobiCom, August 2000.

[12] Y.Dong, W-K Hon, D.K.Y. Yau, J-C Chin, "Distance Reduction in Mobile Wireless Communication;Lower Bound Analysis \& practical Attainment",IEEE transactions on Mobile Computing, vol. 8 no. 2, February 2009.

[13] C.Maihofer, "A survey of geocast routing protocols", IEEE Communications Survey \& Tutorials,vol.6, no.2, Second Quarter 2004.

[14] Young-Bae Ko and Nitin H. Vaidya "Geocasting in Mobile Ad Hoc Networks: Location-Based Multicast Algorithms ", Proceedings of the Second IEEE Workshop on Mobile Computer Systems and Applications (WMCSA), February 1999.

[15] Lynn Choi, Jae Kyun Jung, Byong-Ha Cho and Hyohyun Choi, "M-Geocast: Robust and Energy- Efficient Geometric Routing for Mobile Sensor Networks", SEUS 2008, LNCS 5287,pp. 304-316, IFIP (International Federation for Information Processing) 2008.

[16] A.K.Sadek, W.Yu and K.J.Ray Liu, "On the Energy Efficiency of Cooperative Communications in Wireless Sensor Networks", ACM transactions on Sensor Networks, vol. 6, N0.1: December 2009.

[17] M. Zorzi and R R Rao, "Geographic Random Forwarding(GeRaF) for Adhoc \& Sensor Networks:Energy \& Latency Performance”,IEEE transactions on Mobile Computing, vol.2 no.4, October-December 2003.

[18] A.G. Ruzzelli, G.'Hare and R. Higgs, “Directed Broadcast eith Overhearing for Sensor Networks", ACM Transactions on Sensor Networks, Vol.6, No.1, December 2009. 
International Journal of Wireless \& Mobile Networks (IJWMN) Vol. 4, No. 1, February 2012

Authors

Kaushik Ghosh is an Assistant Professor in the Department of Computer Science \& Engineering, Mody Institute of Technology and Science, Lakshmangargh(Sikar), India. He received his Bachelor of Engineering degree in Electrical \& Electronics Engineering from Sikkim Manipal Institute of Technology and has done his M.Tech in Computer Technology from Jadavpur University, India. His area of research includes Mobile Adhoc Networks (MANETs) and Sensor Networks.

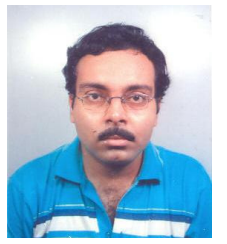

Partha Pratim Bhattacharya received Ph.D (Engg) from Jadavpur University, India in 2007. He has 15 years of experience in teaching and research. At present he is working as Professor in Department of Electronics and Communication Engineering in the Faculty of Engineering and Technology, Mody Institute of Technology and Science (Deemed University), Rajasthan, India. He has published a good number of papers in refereed journals and conferences. His present research interest includes mobile cellular communication, wireless sensor networks and cognitive radio. He is a member of The Institution of Electronics and Telecommunication Engineers, India and The Institution of Engineers, India and received Young Scientist Award from International Union of Radio Science (URSI) in 2005.

Pradip K Das is a Professor and Dean in the Faculty of Engineering \& Technology, Mody Institute of Technology \& Science, Lakshmangargh(Sikar), India. Earlier, he was a Professor and Head in the Department of Computer Science \& Engineering and the founder Director of the School of Mobile Computing \& Communication, Jadavpur University, India. He received his B.E. and M.E. degrees in Electronics and Ph.D (Engg.) in Computer Engineering from Jadavpur University.

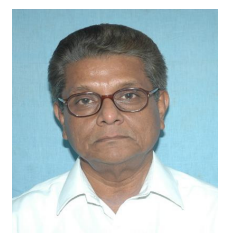

